\title{
The Climate Modelling Toolkit
}

\author{
Joy Merwin Monteiro ${ }^{\ddagger *}$, Rodrigo Caballero*
}

\begin{abstract}
The Climate Modelling Toolkit (CliMT) is a Python-based software component toolkit providing a flexible problem-solving environment for climate science problems. It aims to simplify the development of models of complexity 'appropriate' to the scientific question at hand. This aim is achieved by providing Python-level access to components commonly used in climate models (such as radiative transfer models and dynamical cores) and using the expressive data structures available in Python to access and combine these components. This paper describes the motivation behind developing CliMT, and serves as an introduction to interested users and developers.
\end{abstract}

Index Terms-Climate Modelling, Hierarchical Models

\section{Introduction}

Climate models are numerical representations of the climate system consisting of ocean, land and atmosphere. They have become an important aspect of climate science as they provide a virtual laboratory in which to perform experiments and gain a deeper understanding of the climate system. Climate models can be conceived as a combination of two distinct parts: One, called the "dynamics", is code which numerically integrates the equations of motions of a fluid. The other, called the "physics" is code which approximates various processes considered important for the evolution of the atmospheric/oceanic fluid, including radiation, moist convection and turbulence. Some of these processes, such as convection and turbulence should ideally simulated by the dynamics, but the coarse resolution of typical climate models and limitations of computational resources lead to their being approximated as physics components.

In an influential essay, Isaac Held made the case for studying the climate system using a hierarchy of models, in a manner similar to the hierarchy of model organisms used by evolutionary biologists [Hel05]. The essay argued that such a hierarchy would not only help in our understanding of the climate system, but would also help in interpreting results obtained from more complex models and even aid in improving them. A qualitative description of the climate model ecosystem is shown in Fig. 1. On the dynamics axis, they range from models which represent the atmosphere as a single vertical column to a full turbulent, three dimensional flow. On the physics axis, they range from models which represent radiation or turbulence using ten lines of code to those whose representation of the physics run into thousands of lines.

* Corresponding author: joy.merwin@gmail.com

¥MISU, Stockholm University

Copyright $@ 2016$ Joy Merwin Monteiro et al. This is an open-access article distributed under the terms of the Creative Commons Attribution License, which permits unrestricted use, distribution, and reproduction in any medium, provided the original author and source are credited.
Over the past few decades, efforts have been made to develop such a hierarchy of models (a significant fraction of which has been, not surprisingly, by Isaac's students and collaborators) which have had a positive impact on our understanding of the climate system and the general circulation of the atmosphere [HK81], [HS94], [NZ00], [Sch04], [FHZG06], [MFCE07], [CPM08], [MPFC09]. Note that we restrict our focus here only on numerical models of the climate system, excluding many influential theoretical models such as [HH80] and models of phenomena such as the MaddenJulian Oscillation (MJO) or tropical cyclones.

However, the scale of these efforts has not kept pace with the increasing complexity of full scale general circulation models (GCMs) which are on the threshold of cloud-scale $(\sim 1 \mathrm{~km}$ horizontal resolution) simulations of the entire atmosphere. One of the primary reasons, we believe, is that significant effort is required to build models which represent even the basic features of the atmospheric general circulation. Existing frameworks to develop such models like the Flexible Modelling System (FMS, http://www.gfdl.noaa.gov/fms) and the MITgcm (http://mitgcm.org/) are typically written in Fortran and the effort to set up a model beyond those already provided as examples can, in our experience, be quite discouraging for new users who lack a strong background in Fortran and programming.

In this paper we introduce the Climate Modelling Toolkit (CliMT, pronounced "Klimt"), which attempts to reduce this barrier to developing simplified models of the atmosphere. It is similar in spirit to the above mentioned frameworks, with the following distinctions:

- Configuration and execution of models is much simpler and done in the same script, making repeated simulations less error prone

- New components can be added with minimal infrastructure code requirements, and does not require recompilation of the entire codebase

- Object-oriented design makes program flow both intuitive and less prone to error

- Allows for incremental development: proof of concept development in pure Python and production code in another language (Cython, C, Fortran)

CliMT is currently not capable of parallel execution, and thus is mainly useful for 1 and 2-dimensional climate models. Despite these limitations, CliMT is used by around 10 research groups around the world (based on user queries/feedback) for research [CPM08], [CH13], [RBSB10] and pedagogy [Pie10]. In the following sections, we describe the basic building blocks of CliMT, their usage, and how new components can be added. We end with a roadmap towards version 1.0 of CliMT, which should 


\begin{tabular}{|c|l|}
\hline Physical Process & Components \\
\hline Convection & Zhang-McFarlane \\
& Emanuel \\
& Emanuel hard adjustment \\
& Simplified Betts-Miller \\
\hline Dynamics & Axisymmetric dynamics \\
& Two column dynamics \\
\hline Ocean & Slab Ocean \\
\hline Radiation & Community Atmosphere Model (CAM) 3 \\
& CCM 3 \\
& Chou \\
& Grey Gas \\
& Rapid Radiative Transfer Model (RRTM) \\
& Insolation \\
& Absorption by ozone \\
\hline Turbulence & $\begin{array}{l}\text { CCM3 } \\
\text { Simple (diffusive) }\end{array}$ \\
\hline Thermodynamics & routines for calculating thermodynamic quantities \\
\hline
\end{tabular}

TABLE 1: Components available currently in CliMT.

see CliMT working as a fully parallel, moist GCM capable of simulating a realistic climate.

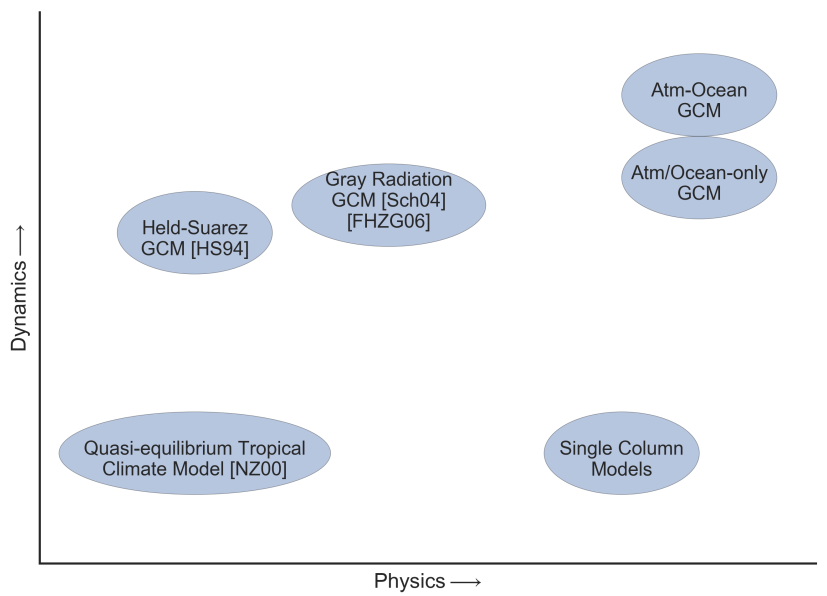

Fig. 1: A qualitative depiction of the climate model hierarchy. The complexity of the dynamics and the physics models increases along the direction of the arrows. This is merely an indicative representation, and is not meant to be exhaustive.

\section{CliMT: best of both worlds}

CliMT combines the elegance and clarity of the Python language with the efficiency of Fortran libraries. Users interact with CliMT in a pythonic way, using high-level data structures like dictionaries and lists, and the numerical computations are done by optimised (and tested) Fortran code extracted from state-of-the-art climate models. Currently, f2py is used to convert Fortran code to a library that can be imported into Python. Table 1 lists the physical processes that can be currently simulated using CliMT and the options available to represent each physical process.

The initialization of the components and the execution of the resulting model is handled in the same script, which makes the parameters and assumptions underlying the model explicit. This makes interpreting the results of the simulation easier. Given that model initialization, execution and data analysis can be performed from within a single IPython notebook, this makes model results and the resulting scientific results reproducible as well. CliMT also enables users to study the effects of changing physical parameterizations and dynamical cores on the simulated climate, something that is difficult to do in other idealised modelling frameworks.

\section{Architecture}

CliMT, in a broad sense, is a library which enables numerical representations of different processes in the climate system to be linked together in an intuitive manner. While it provides a leapfrog integrator (a second order method for numerical integration common to many climate models) to step the model forward in time, it does not provide routines to calculate gradients or spectral coefficients. All components in CliMT are either written from scratch or extracted from larger climate models (especially radiative transfer models). There is no facility to update the underlying Fortran/C code itself if the original code is updated. It is assumed that each component will implement any numerical methods that it requires. While this may lead to some code duplication, it allows for a loose coupling between the various components. This allows development of new components without recompilation of the entire codebase.

When a component is instantiated, CliMT queries the component to find out which variables the component affects. For instance, a convection component will affect the specific humidity and the temperature variables. It creates a Numpy array of the appropriate dimensions for each such variable. If multiple components affect the same variable, only one such array is created. During execution, it collects the time tendency ${ }^{*}$ terms from each component (in the form of a Numpy array), sums them together and uses the resulting cumulative tendency to step the model forward in time. Currently, it is assumed that all components share a common grid, i.e, all arrays representing tendency terms have the same shape, and represent the same location in three dimensional space. As is commonly the case in climate models, the spatial coordinates are in latitude-longitude-pressure space, and CliMT does a sanity check to ensure that all components have the same spatial representation (i.e, tendency arrays expected from each component has the same shape).

To summarize, each component (encapsulated in the Component class) provides time tendency terms to the main execution loop, and the model is stepped forward in time by integrating these tendencies using the leapfrog integrator. Optionally, the model state is displayed using a wrapper over matplotlib and written to disk using the netCDF 4 library. Since the model state variables are Numpy arrays, they can be easily accessed by external Python libraries for online processing or any other purpose.

Combining multiple Component objects is made possible using the Federation class. Combining two or more desired Component objects in a Federation results in a climate model of appropriate complexity.

The Component and Federation classes are the interface between the end-user and CliMT, with all other classes being used internally by these two classes.

*. A time tendency term at time $t_{1}$ is the incremental value of a variable to be added to obtain that variable's value at time $t_{2}$, where $t_{2}$ is the time instant succeeding $t_{1}$. 


\section{Component}

A Component class is the fundamental abstraction in CliMT. It encapsulates the behavior of a component that takes certain inputs and provides certain tendencies as output. Each Component object has (among others) the following members which are specified by the developer:

- Prognostic

- Diagnostic

- Fixed

- Fromextension

- ToExtension

These members are lists whose elements are one of many predefined field names (available in the state class) relevant to climate science applications. For example, if Component.Prognostic = ['U', ' $\mathrm{V}$ ', ' theta'], then the component represents a model which can forecast the future state of the wind along longitude, wind along latitude and the potential temperature, respectively. The Diagnostic list contains those fields which the component calculates using the prognostic fields, and the Fixed list contains those fields which are left unchanged by the component. The ToExtension list indicates which fields are required by the component to forecast the future state, and the FromExtension list indicates which fields are returned by the component. Typically, the FromExtension list contains the name of fields with an Inc suffix, indicating that the component returns increments only, which are to be stepped forward in time. The term Extension refers to the compiled Fortran/C library which does the actual computation. Each Component also keeps track of the time step dt taken during each integration (normally decided by stability constraints), and the time elapsed from the beginning of the integration.

Component has two main methods: compute and step. The compute method calls the compiled Fortran/C code and retrieves the increments and diagnostic fields and stores them internally. compute takes an optional boolean argument ForcedCompute. If ForcedCompute is true, then the tendency terms are always calculated. If it is false (the default), then the tendencies are calculated only if the elapsed time is at least $d t$ greater than the previous time at which the tendencies were calculated. Such behavior is required when combining two components which operate on very different time scales, such as convection (time scale of hours) and radiation (time scale of days). compute is also invoked by simply calling the object.

The step method steps the component forward in time by taking the increments calculated in compute and passing them on to the leapfrog integrator (available in the infrastructure code, not in each individual component) to get future values of the fields. step internally calls compute, so the user needs only to call step. step accepts two optional arguments Inc and RunLength. Inc which is a dictionary whose keys are some or all of the elements in ToExtension, and the corresponding values are additional tendency terms calculated outside the component. These increments are added to the internally computed tendency terms before calling the integrator. Runlength decides how many seconds forward in time the component is stepped forward. If RunLength is a positive integer, then the component is stepped forward in time RunLength $* d t$ seconds. If it is a positive floating point number, then the component is stepped foward in time RunLength seconds.
All parameters required by any Component are passed as a dictionary during object instantiation. This includes initial values of the fields integrated by the Component. If no initial values are supplied, the fields are initialized as zeroed NumPy arrays of the appropriate shape. An example which uses the CAM radiative transfer model to compute the radiative tendencies is shown below (also available in the source code itself):

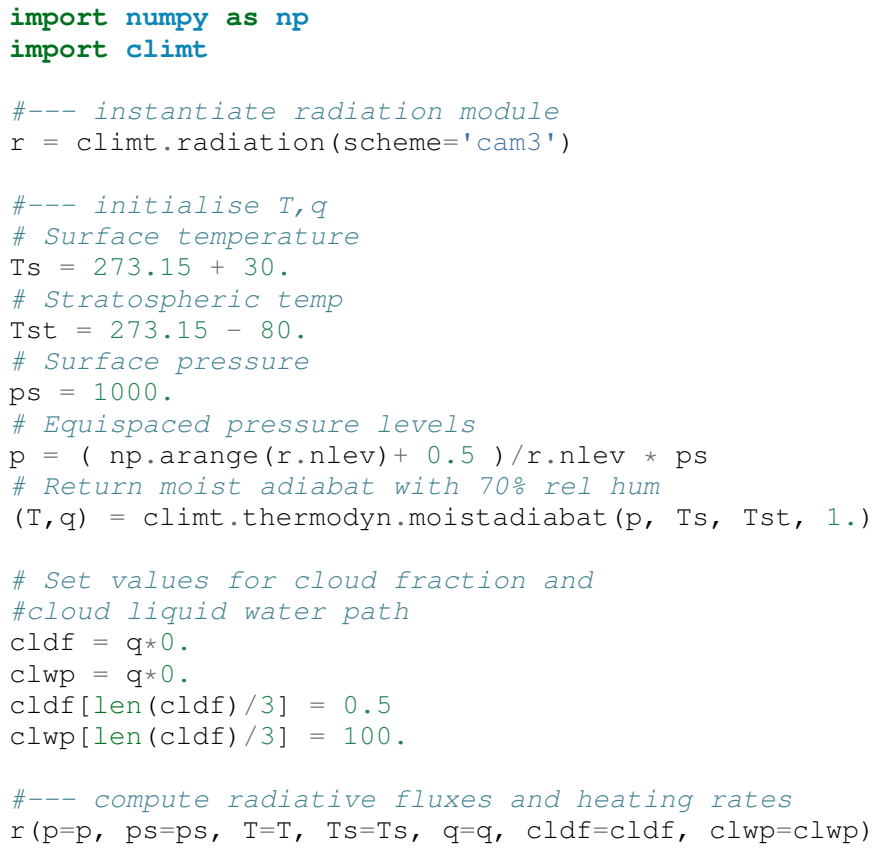

In the above code, the computed outputs can be accessed by treating $r$ as a dictionary: the shortwave flux at the top of the atmosphere is available at $r$ [ 'SwToa' ], for example.

\section{Federation}

Federation is a subclass of Component which is instantiated by providing two or more Component objects as arguments. It provides the same interface as Component, and is the abstraction of a climate model with multiple interacting components. On instantiation, Federation does a few sanity checks to ensure consistency of dimensions between its member Components. As in Component, integrating the Federation forward in time is simply achieved by calling step. An example which computes the radiative convective equilibrium in a column of the atmosphere is given below:

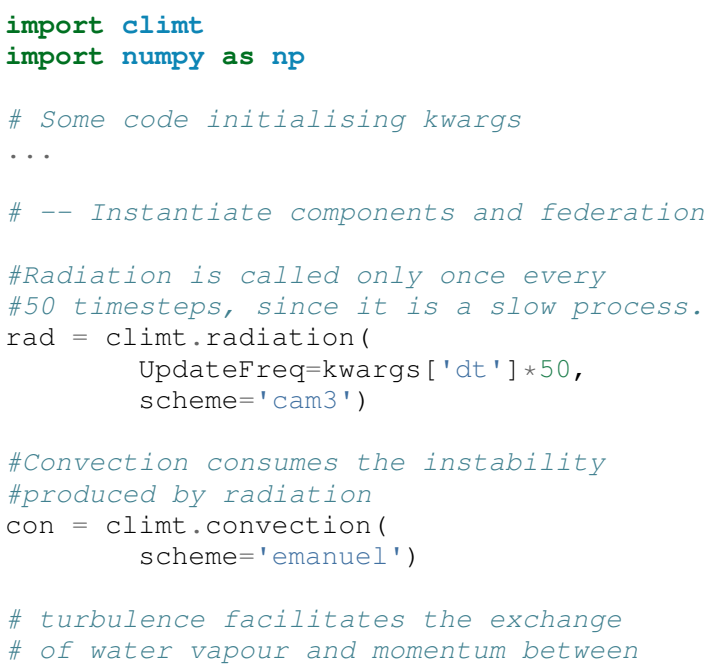


\# the ocean and the atmospheric column

dif $=$ climt.turbulence ()

\#ocean provides a source of water vapour

oce $=$ climt.ocean ()

\#Instantiate the federation

fed = climt.federation(dif, rad, oce, con, $\star \star k$ kwargs )
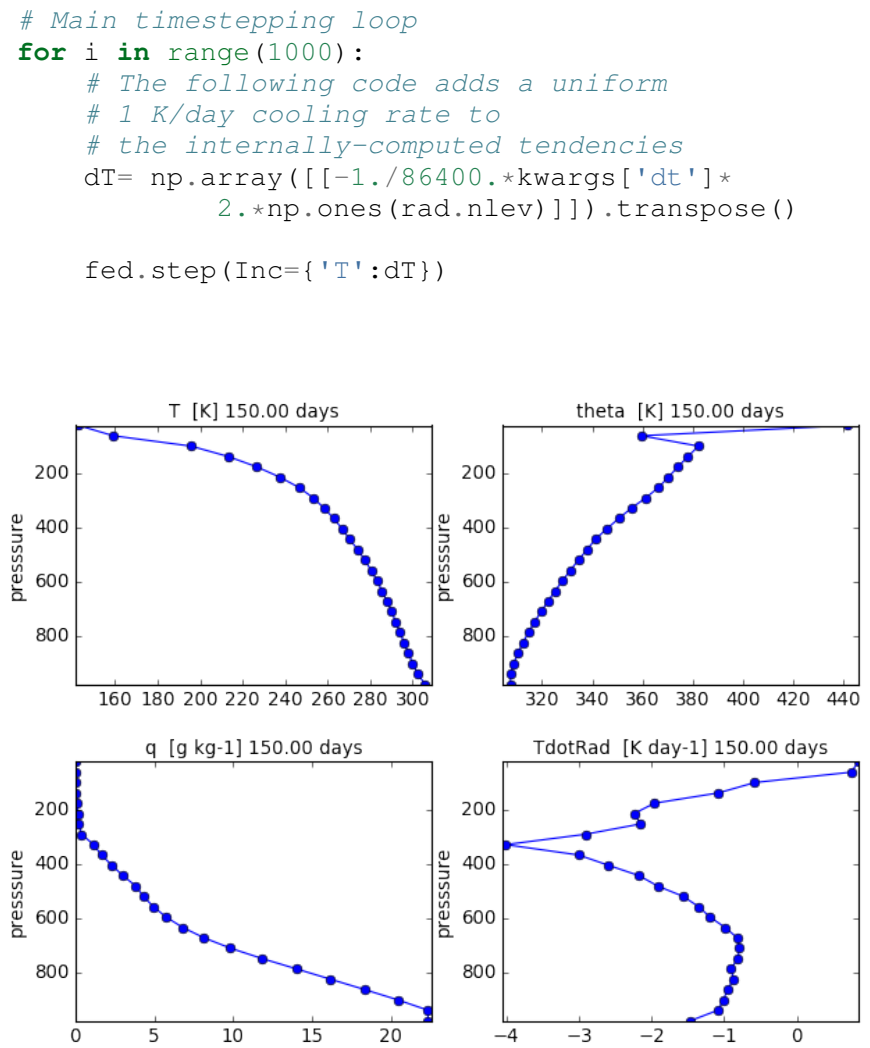

Fig. 2: The displayed output from a one dimensional (vertical) radiative-convective simulation on day 150. The fields are updated in real time during the simulation. The panels display (clockwise from top left): Temperature, Potential Temperature, radiative heating and specific humidity respectively. The y axis is height measured in pressure and has units of millibar (100 Pascals $=1$ millibar $)$. As expected from theory and observations, the temperature decreases almost linearly in the lower levels of the column.

Here, the radiative code has an UpdateFreq value that is 50 times the actual timestep of the federation. As mentioned before, this feature facilitates coupling of components whose characteristic time scales are very different from each other without increasing the computational load during the simulation. Notice also the external tendency term dT passed on to fed in the step method. The output fields are again accessed by treating fed as a dictionary. Figure 2 shows the typical output from a CliMT radiative-convective simulation; Display and I/O is discussed in the next section.

\section{Software Layout and Documentation}

CliMT maintains the infrastructure code and the actual component code in separate folders. The src directory contains the component code whereas lib/climt contains the infrastructure code. The main infrastructure code resides in \{component, federation, state, grid\}.py. The various physical processes are accessible from appropriately named files in lib/climt (e.g, convection.py). These files implement the Component class and act as an interface to the underlying Fortran code. Note that there is no restriction on the language in which extensions are written. All the physical variables that CliMT recognises are listed in state.py. While all files themselves have detailed inline documentation, there is currently no automated system in place to build a module reference based on these comments. Querying an object in an IPython environment is currently the best way of accessing the documentation, as demonstrated in Fig. 3. Addition of a new module would require copying the extension code to $\mathrm{src} /$, adding a reference to it in the appropriate physical process file (e.g, a new dynamical core would be included in dynamics.py), and adding a reference in setup.py to enable building and installation.

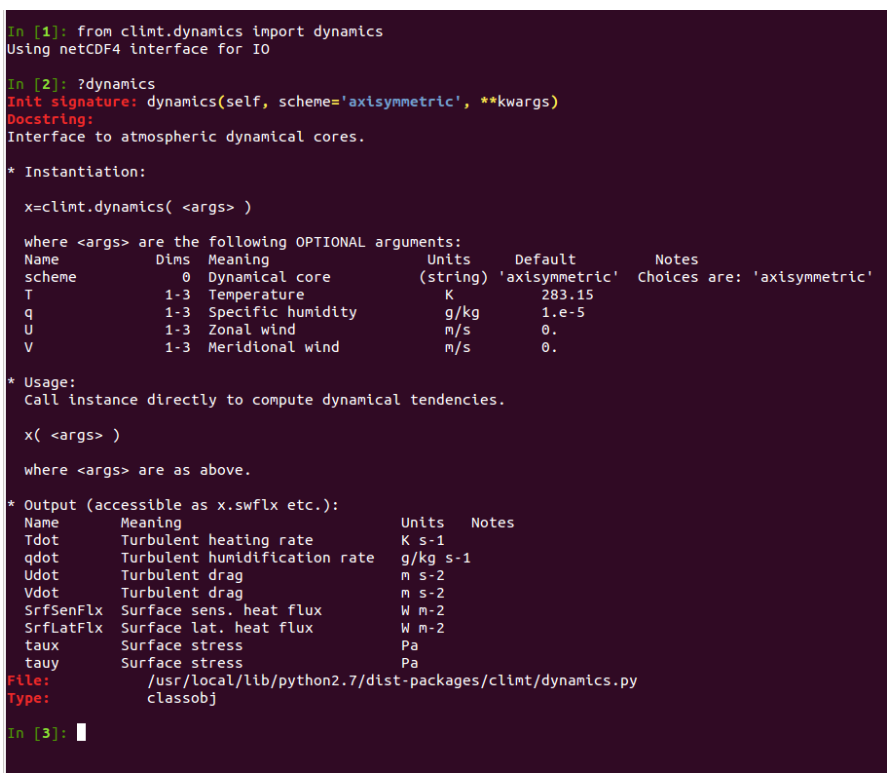

Fig. 3: Accessing documentation for the dynamics class in an IPython prompt.

\section{Monitoring fields and $\mathrm{I} / \mathrm{O}$}

CliMT also provides for real time display (monitoring) of the simulated fields. Currently, up to four fields can be monitored. Monitoring is activated by providing an additional argument during component instantiation called MonitorFields. Monitorfields is a list of up to four fields that are part of the simulation. If the field is three dimensional, the zonal average (average along longitude) is displayed. The frequency at which the display is refreshed is decided by the MonitorFreq argument.

CliMT can read intial conditions from the file whose name is specified in the RestartFile argument. The output is written to the file whose name is specified in the Output File argument. If RestartFile and OutputFile are the same, then the data is appended to OutputFile. The last time slice stored in RestartFile is used to initialize the model. If some fields are missing in RestartFile, they are initialized to default (zero) values.

The fields written to the output file are specified in the OutputFields argument. If OutputFields is not specified, all fields are written to file. OutputFreq is an optional argument 
which specifies the time between writing data to file. If it is not specified, the output is stored once every model day.

\section{Developing new Components}

CliMT requires a single point of entry into the Fortran/C code to be provided by each Component: the driver method. The driver method takes as input NumPy arrays representing the fields required to calculate the tendency terms. The order in which the fields are input is represented by the ToExtension list in the Component. The output of the driver is a list of NumPy arrays ordered in the same way as the FromExtension list. The translation between NumPy arrays and the Fortran code is currently done automatically by $\mathrm{f} 2 \mathrm{py}$ generated code. The Fortran/C extension module itself is stored in Component.Extension and an optional name is provided in Component. Name. Component.Required is a list of those fields which are essential for the component to calculate tendencies. These variables along with Prognostic, Diagnostic and Fixed lists (which were previously discussed) enable CliMT to interface with a new component.

We note that CliMT expects the tendency terms to be premultiplied by dt, i.e, the units of the fields returned by driver is expected to be the same as the units of the prognostic fields. The integrator does not multiply the tendency terms by $d t$, as is normally the case.

\section{Current Development: towards CliMT 1.0}

The space occupied by CliMT in the climate model hierarchy is shown in Fig. 4. It is currently capable of simulating relatively simple (1 and 2 dimensional) dynamics and quite sophisticated physical processes. Moving forward, we hope fulfill the vision of using CliMT as a full fledged moist idealized GCM. As a first step, we have integrated a dynamical core adapted from the Global Forecast System (GFS). Together with this, we have added a new Held-Suarez module which provides the Held-Suarez forcing terms for a 3-d atmosphere. A working example of the benchmark is now available from a development fork (available at https://github.com/JoyMonteiro/CliMT/lib/ examples). Figure 5 shows the mean wind along longitudes ("zonal" wind) simulated by the model. It shows most of the important aspects of the mean circulation in the earth's atmosphere: strong westerly jet streams around 30 degrees N/S and easterly winds near the surface and the top of the atmosphere in the tropics.

Many changes were incorporated enroute this integration. The dynamical core is the first component of CliMT that interfaces with the Fortran library using Cython and the ISO_C_Binding module introduced in Fortran 2003. This will be used as a template to eventually move all components to a Cython interface: $\mathrm{f} 2 \mathrm{py}$ does not seem to be actively developed anymore, and currently cannot interface with code that includes compound data structures, like the FMS dynamical cores. Therefore, we expect the CythonISO_C_Binding combination to enable CliMT to use a wider range of libraries.

A new feature in CliMT 1.0 will be to allow components to use an internal integrator and not the default leapfrog available in CliMT. This is useful since components such as the 3-D dynamical core already include non-trivial implementations of numerical integrators which will have to be reimplemented in CliMT to ensure stable integrations. Moreover, it is unlikely that atmosphere

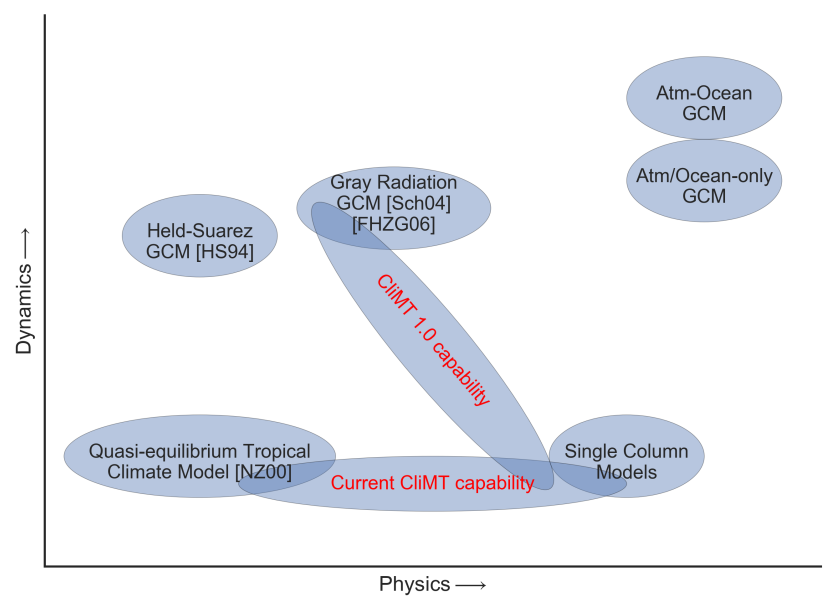

Fig. 4: A look at the current capability and future directions for CliMT development in context of the model hierarchy

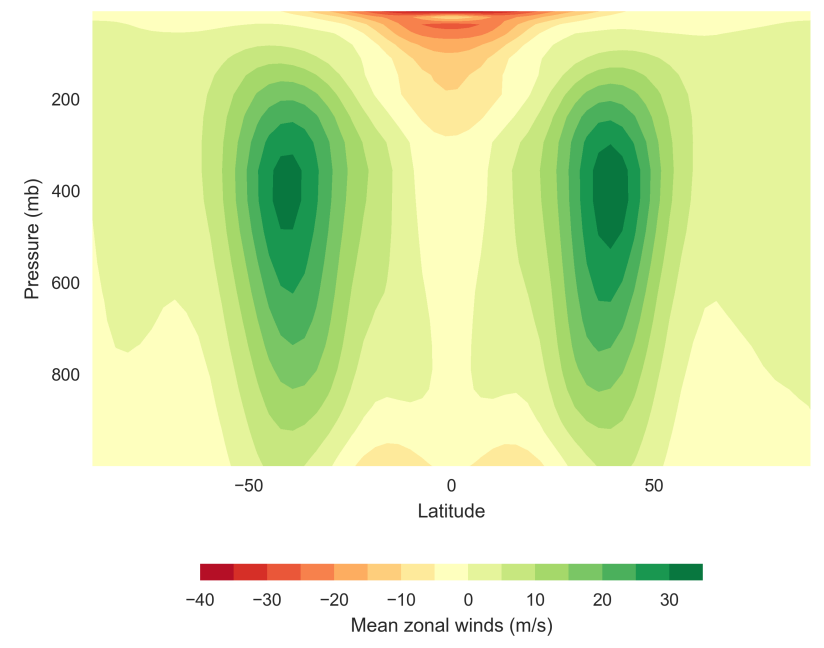

Fig. 5: The mean wind along longitudes in the Held-Suarez, simulation. The mean is over 1000 days and over all longitudes (a "zonal" mean). The y-axis has units of millibar $(=100 \mathrm{~Pa})$. It compares well with the simulated winds in [HS94] (see Fig. 2 in their paper)

and ocean models use similar numerical algorithms. Since the focus of CliMT is on the infrastructure and not the numerics, this feature enables rapid addition of new components into CliMT without substantially changing its basic structure. This feature and other enhancements will be described in detail in a forthcoming paper accompanying the release of CliMT 1.0.

Next, we intend to interface the dynamical core with the grey gas radiation module to enable CliMT to generate a realistic general circulation without using the Held-Suarez forcing. Issues we intend to address in the future include:

- $\quad$ scalability by making CliMT MPI and/or OpenMP-aware

- More systematic testing architecture

- A full user manual and IPython notebook examples

With these additions, we hope CliMT will be the framework of choice for a wide audience, from undergraduates to scientists to explore questions in climate science.

\section{Acknowledgements}

This work is supported by funding from the Swedish e-Science Research Centre (http: //www.e-science.se/). 


\section{REFERENCES}

[CH13] R. Caballero and M. Huber. State-dependent climate sensitivity in past warm climates and its implications for future climate projections. Proceedings of the National Academy of Sciences, 110(35):14162-14167, August 2013.

[CPM08] R. Caballero, R. T. Pierrehumbert, and J. L. Mitchell. Axisymmetric, nearly inviscid circulations in non-condensing radiativeconvective atmospheres. Quarterly Journal of the Royal Meteorological Society, 134(634):1269-1285, July 2008.

[FHZG06] D. M. W. Frierson, I. M. Held, and P. Zurita-Gotor. A GrayRadiation Aquaplanet Moist GCM. Part I: Static Stability and Eddy Scale. Journal of the Atmospheric Sciences, 63(10):25482566, October 2006.

[Hel05] I. M. Held. The Gap between Simulation and Understanding in Climate Modeling. Bulletin of the American Meteorological Society, 86(11):1609-1614, November 2005.

[HH80] I. M. Held and A. Y. Hou. Nonlinear Axially Symmetric Circulations in a Nearly Inviscid Atmosphere. Journal of the Atmospheric Sciences, 37(3):515-533, March 1980.

[HK81] B. J. Hoskins and D. J. Karoly. The steady linear response of a spherical atmosphere to thermal and orographic forcing. Journal of the Atmospheric Sciences, 38(6):1179-1196, 1981.

[HS94] I. M. Held and M. J. Suarez. A Proposal for the Intercomparison of the Dynamical Cores of Atmospheric General Circulation Models. 75(10):1825-1830, October 1994.

[MFCE07] J. Marshall, D. Ferreira, J-M. Campin, and D. Enderton. Mean Climate and Variability of the Atmosphere and Ocean on an Aquaplanet. Journal of the Atmospheric Sciences, 64(12):4270 4286, December 2007.

[MPFC09] J. L. Mitchell, R. T. Pierrehumbert, D. M.W. Frierson, and R. Caballero. The impact of methane thermodynamics on seasonal convection and circulation in a model Titan atmosphere. Icarus, 203(1):250-264, September 2009.

[NZ00] J. D. Neelin and N. Zeng. A Quasi-Equilibrium Tropical Circulation Model-Formulation. Journal of the atmospheric sciences, 57(11):1741-1766, 2000

[Pie10] R. T. Pierrehumbert. Principles of planetary climate. Cambridge University Press, 2010.

[RBSB10] M. T. Rosing, D. K. Bird, N. H. Sleep, and C. J. Bjerrum. No climate paradox under the faint early Sun. Nature, 464(7289):744747, April 2010.

[Sch04] T. Schneider. The Tropopause and the Thermal Stratification in the Extratropics of a Dry Atmosphere. Journal of the Atmospheric Sciences, 61(12):1317-1340, June 2004. 